Bull. Austral. Math. Soc.

Vol. 48 (1993) [75-91]

\title{
THE IMPLICATIONS FOR DIFFERENTIABILITY OF A WEAK INDEX OF NON-COMPACTNESS
}

\author{
John R. Giles and Warken B. Moors
}

\begin{abstract}
In a recent paper the authors showed that certain set-valued mappings from a Baire space into subsets of a Banach space which have a continuity property defined in terms of Kuratowski's index of non-compactness have inherent single-valued properties. Here we generalise the continuity property to one defined in terms of a weak index of non-compactness and we show that this wider class of setvalued mappings also has significant implications for the differentiability of convex functions on Banach spaces.
\end{abstract}

\section{INTRODUCTION}

For a bounded set $E$ in a metric space $X$, the Kuratowski index of non-compactness of $E$ is $\alpha(E) \equiv \inf \{r: E$ is covered by a finite family of sets of diameter less than $r\}$. In [7], we investigated the special properties of a new continuity property defined in terms of Kuratowski's index of non-compactness. A set-valued mapping $\Phi$ from a topological space $A$ into subsets of a metric space $X$ is called $\alpha$ upper semi-continuous at $t \in A$ if given $\varepsilon>0$, there exists an open neighbourhood $U$ of $t$ such that $\alpha(\Phi(U))<\varepsilon$. We showed that a minimal weak* upper semi-continuous compact convex set-valued mapping from a Baire space into subsets of the dual of a Banach space which is $\alpha$ upper semi-continuous on a dense subset of its domain is generically single-valued. This has consequences in particular for differentiability theory for convex functions of Banach spaces.

Here we study a similar continuity property defined in terms of a weaker index of non-compactness introduced by de Blasi, [4]. We consider a Banach space $X$ over the real numbers with dual $X^{*}$ and denote the closed unit ball of $X$ by $B(X) \equiv\{x \in$ $X:\|x\| \leqslant 1\}$ and the unit sphere of $X$ by $S(X) \equiv\{x \in X:\|x\|=1\}$. For a bounded set $E$ in $X$ the weak index of non-compactness of $E$ is

$\omega(E) \equiv \inf \{r:$ there exists a weakly compact set $C$ such that $E \subseteq C+r B(X)\}$

Received 19 August 1992

We would like to thank Denka Kutzarova and Pier Papini for drawing our attention to de Blasi's $\omega$ index of non-compactness.

Copyright Clearance Centre, Inc. Serial-fee code: $0004-9729 / 93 \quad \$ A 2.00+0.00$. 
This $\omega$ index has the elementary properties satisfied by Kuratowski's $\alpha$ index for bounded subsets of a Banach space, $[1$, p.216]:

(i) $\omega(E)=0$ if and only if $E$ is relatively weakly compact,

(ii) if $E \subseteq F$ then $\omega(E) \leqslant \omega(F)$,

(iii) $\omega(k E)=|k| \omega(E)$, for each real $k$,

(iv) $\omega(\overline{c o} E)=\omega(E)$, where $\overline{c o} E$ denotes the closed convex hull of $E$. The $\omega$ index also satisfies a similar generalisation of Cantor's intersection property:

(v) given a nested sequence of non-empty weakly closed sets $\left\{F_{n}\right\}, F_{1} \supseteq$ $F_{2} \supseteq \ldots \supseteq F_{n} \supseteq \ldots$ with the property that $\lim _{n \rightarrow \infty} \omega\left(F_{n}\right)=0$ then $\bigcap_{1}^{\infty} F_{n}$ is non-empty and weakly compact.

We say that a set-valued mapping $\Phi$ from a topological space into subsets of a Banach space $X$ is $\omega$ upper semi-continuous at $t \in A$ if given $\varepsilon>0$, there exists an open neighbourhood $U$ of $t$ such that $\omega(\Phi(U))<\varepsilon$. In Section 2 we examine how this property is related to the usual norm and weak upper semi-continuity properties for set-valued mappings. We show that any minimal weak* cusco from a Baire space into subsets of the dual of a Banach space which is $\omega$ upper semi-continuous on a dense subset of its domain is single-valued and norm upper semi-continuous on a dense $G_{\delta}$ subset of its domain.

Given a non-empty bounded set $E$ in a Banach space $X$, for $f \in X^{*} \backslash\{0\}$ and $\delta>0$, the slice of $E$ defined by $f$ and $\delta$ is the subset $S(E, f, \delta) \equiv\{x \in F: f(x)>$ $\sup f(E)-\delta\}$. In Section 3 we show that for closed bounded convex sets in any Banach space, certain slices of small $\omega$ index contain slices of small diameter and this enables us to establish an equivalence of denting point structure for denting points defined by diameter and $\omega$ index.

A Banach space is called an Asplund space if every continuous convex function on an open convex domain is Fréchet differentiable on a dense $G_{\delta}$ subset of its domain. Namioka and Phelps, [13, p.739] gave the main characterisation theorem for such spaces in terms of weak* strongly exposed point structure of weak* compact convex sets in the dual. In Section 4, we extend this characterisation theorem using the $\omega$ index. A recent result, [9, Theorem 3.5], established special differentiability properties for those Banach spaces which have an equivalent norm where every point of the unit sphere is a denting point. We extend this result to show that the same differentiability properties hold for those Banach spaces which have an equivalent norm where every point of the unit sphere is an $\omega$ denting point.

\section{THE SPECIAL PROPERTIES OF $\omega$ UPPER SEMI-CONTINUOUS MAPPINGS}

A set-valued mapping $\Phi$ from a topological space $A$ into subsets of a topological 
space $X$ is said to be upper semi-continuous at $t \in A$, if given an open subset $W$ containing $\Phi(t)$ there exists an open neighbourhood $U$ of $t$ such that $\Phi(U) \subseteq W$. We call such a $\Phi$ an usco mapping at $t$ if also $\Phi(t)$ is non-empty and compact; when $X$ is a linear topological space we call such a $\Phi$ a cusco at $t$ if $\Phi(t)$ is non-empty, convex and compact.

For set-valued mappings from a topological space into a Banach space there is a very satisfying close relation between $\alpha$ upper semi-continuity and norm upper semicontinuity, [7, Theorem 2.4]. The relation between $\omega$ upper semi-continuity and norm and weak upper semi-continuity is not quite so straightforward.

Theorem 2.1. Consider a set-valued mapping $\Phi$ from a topological space $A$ into subsets of a Banach space $X$.

(i) If $\Phi$ is norm upper semi-continuous at $t \in A$ and $\Phi(t)$ is weakly compact then $\Phi$ is $\omega$ upper semi-continuous at $t$.

(ii) If $\Phi$ is $\omega$ upper semi-continuous at $t \in A$ and $\Phi(t)=\cap\left\{\overline{\Phi(U)}^{w}: U \in \mathcal{B}\right\}$ where $\mathcal{B}$ is a local base for $t$ then $\Phi$ is weak upper semi-continuous at $t$ and $\Phi(t)$ is weakly compact.

Proof: (i) If $\Phi$ is norm upper semi-continuous at $t \in A$, then, given $\varepsilon>0$ there exists a neighbourhood $U$ of $t$ such that $\Phi(U) \subseteq \Phi(t)+\varepsilon B(X)$. If $\Phi(t)$ is weakly compact then this implies that $\omega(\Phi(U))<\varepsilon$.

(ii) If $\Phi$ is $\omega$ upper semi-continuous at $t \in A$ there exists a nested sequence $\left\{U_{n}\right\}$ of open neighbourhoods of $t$ such that $\lim _{n \rightarrow \infty} \omega\left(\Phi\left(U_{n}\right)\right)=0$. So by $\omega$ property (iv), $\lim _{n \rightarrow \infty} \omega{\overline{\left(\Phi\left(U_{n}\right)\right)}}^{w}=0$ and by $\omega$ property (v), $\bigcap_{1}^{\infty}{\overline{\Phi\left(U_{n}\right)}}^{w}$ is weakly compact. But $\bigcap_{1}^{\infty}{\overline{\Phi\left(U_{n}\right)}}^{w} \supseteq \cap\left\{\overline{\Phi(U)}^{w}: U \in \mathcal{B}\right\}=\Phi(t)$, so $\Phi(t)$ is also weakly compact. For a weakly open set $W$ where $\Phi(t) \subseteq W$, consider $K \equiv \bigcap_{1}^{\infty} \overline{\Phi\left(U_{n}\right)}{ }^{w} \backslash W$ which is weakly compact. Now $\cap\left\{\overline{\Phi(U)}^{w}: U \in \mathcal{B}\right\} \cap K=\Phi(t) \cap K=\emptyset$ so by the finite intersection property there exists a finite subfamily $\left\{U_{\alpha_{1}}, \ldots, U_{\alpha_{n}}\right\}$ in $\mathcal{B}$, such that $\emptyset=\bigcap_{1}^{n}{\overline{\Phi\left(U_{\alpha_{i}}\right)}}^{w} \cap K=$ ${\overline{\Phi\left(\bigcap_{1}^{n} U_{\alpha_{i}}\right)}}^{w} \cap K$. So there exists some $V \in \mathcal{B}$ such that $\overline{\Phi(V)}^{w} \cap K=0$. Now $M \equiv \overline{\Phi(V)}^{w} \backslash W$ is weakly closed. If for each $n \in \mathbb{N},{\overline{\Phi\left(U_{n}\right)}}^{w} \cap M \neq \emptyset$ then by $\omega$ property (v), $\emptyset \neq \bigcap_{1}^{\infty}{\overline{\Phi\left(U_{n}\right)}}^{w} \cap M=\overline{\Phi(V)}^{w} \cap K$. So there exists some $n_{0} \in \mathbb{N}$ such that $\overline{\Phi\left(U_{n_{0}}\right)}{ }^{w} \cap M=\emptyset$. But then $\Phi\left(U_{n_{0}} \cap V\right) \backslash W \subseteq \overline{\Phi\left(U_{n_{0}}\right)}{ }^{w} \cap \overline{\Phi(V)}{ }^{w} \backslash W=\emptyset$. So we conclude that there exists some $U \in \mathcal{B}$ such that $\Phi(U) \subseteq W$; that is $\Phi$ is weakly upper semi-continuous at $t$. 
We should note that the local base condition given in Theorem 2.1(ii) is always fulfilled by a set-valued mapping which is weak upper semi-continuous and weakly compact valued, (see [7, Proposition 2.1]).

A set valued mapping which is $\omega$ upper semi-continuous is not necessarily norm upper semi-continuous as the following example shows. Consider $X$ an infinite dimensional reflexive Banach space and the identity mapping id from $B(X)$ with the relative weak topology into $X$ with its norm topology. Now id is clearly not norm upper semi-continuous on $B(X)$, but since $B(X)$ is weakly compact, it is trivially $\omega$ upper semi-continuous on $B(X)$.

After Theorem 2.4 we shall give an example to show that there is no neat equivalence of $\omega$ upper semi-continuity with weak upper semi-continuity.

The significance of this generalised continuity property we have introduced is that an important class of such mappings from a Baire space are single-valued on a dense $G_{\delta}$ subset of their domain. This is a consequence of the special properties of weakly compact convex sets in Banach spaces which were pointed out by Bourgain, [2].

We need to examine more closely the definition of $\omega$ upper semi-continuity.

LEMma 2.2. Given a non-empty subset $E$ of a Banach space $X$ and $r>0$, if $E \subseteq C+r B(X)$ where $C$ is a weakly compact subset of $X$ then there exists a minimal convex weakly compact subset $C_{m}$ such that $E \subseteq C_{m}+r B(X)$.

Proof: Let $\mathcal{P}$ denote the family of non-empty convex weakly compact subsets $C$ of $X$ such that $E \subseteq C+r B(X)$. Now $\mathcal{P}$ is non-empty since $\overline{c o} C$ is weakly compact, [3, p.68], and $E \subseteq \overline{c o} C+r B(X)$. Consider $\mathcal{P}$ partially ordered by set inclusion and $\left\{C_{\alpha}\right\}$ a totally ordered subfamily of $\mathcal{P}$. Clearly $\bigcap_{\alpha} C_{\alpha}$ is non-empty convex and weakly compact, and for each $x \in E,(x+r B(X)) \cap C_{\alpha} \neq \emptyset$ for all $\alpha$, so by the finite intersection property $(x+r B(X)) \cap \bigcap_{\alpha} C_{\alpha} \neq \emptyset$ so $E \subseteq \bigcap_{\alpha} C_{\alpha}+r B(X)$; that is, $\bigcap_{\alpha} C_{\alpha} \in \mathcal{P}$. And $\bigcap_{\alpha} C_{\alpha} \subseteq C_{\alpha}$ for all $\alpha$. Therefore, by Zorn's Lemma, $\mathcal{P}$ has a minimal element $C_{m}$.

An usco (cusco) mapping from a topological space into subsets of a topological space (linear topological space) is said to be minimal if its graph does not contain the graph of any other usco (cusco) with the same domain. Given a minimal weak* usco $\Phi$ from a topological space $A$ into subsets of the dual $X^{*}$ of a Banach space $X$, it is known that the mapping $\bar{\Phi}$ defined by $\bar{\Phi}(x)=\overline{\mathrm{co}}^{w^{*}} \Phi(x)$ is a minimal weak* cusco from $a$ into subsets of $X^{*},[8$, p.357]. So throughout the paper we work with minimal cuscos keeping in mind that results extend to minimal uscos by this relation.

We need the following characterisation of minimal cuscos, [7, Lemma 2.5].

Proposition 2.3. A cusco $\Phi$ from a topological space $A$ into subsets of a 
separated linear topological space $X$ is a minimal cusco if and only if for any open set $V$ in $A$ and closed convex set $K$ in $X$ where $\Phi(V) \nsubseteq K$ there exists a non-empty open set $V^{\prime} \subseteq V$ such that $\Phi\left(V^{\prime}\right) \cap K=\emptyset$.

Theorem 2.4. Consider a topological space $A$ and a Banach space $X$. We denote by $\tau$ the norm or weak topology on $X$ and if $X$ is the dual of a Banach space then also the weak ${ }^{*}$ topology on $X$. Consider a minimal $\tau$-cusco $\Phi$ from $A$ into-subsets of $X$.

(i) Given any non-empty open subset $V$ of $A$ and $\varepsilon>0$ such that $\omega(\Phi(V))<$ $\varepsilon / 4$, there exists a non-empty open subset $U$ of $V$ such that $\operatorname{diam} \Phi(U)<$ $\varepsilon$.

(ii) If $A$ is a Baire space and $\Phi$ is $\omega$ upper semi-continuous on a dense subset of $A$ then $\Phi$ is single-valued and norm upper semi-continuous on a dense $G_{\delta}$ subset of $A$.

Proof: (i) By Lemma 2.2 there exists a minimal convex weakly compact set $C_{m}$ such that $\Phi(V) \subseteq C_{m}+(\varepsilon / 4) B(X)$. We assume that $\operatorname{diam} C_{m} \geqslant \varepsilon / 2$. Since $C_{m}$ is weakly compact and convex there exist $f \in S\left(X^{*}\right)$ and $\delta>0$ such that $\operatorname{diam} S\left(C_{m}, f, \delta\right)<\varepsilon / 2,\left[2\right.$, p.199]. Now $K \equiv C_{m} \backslash S\left(C_{m}, f, \delta\right)$ is weakly compact and convex and so it is $\tau$-closed and convex. But $K+(\varepsilon / 4) B(X)$ is also $\tau$-closed and convex. Since $C_{m}$ is a minimal convex weakly compact set, $\Phi(V) \nsubseteq K+(\varepsilon / 4) B(X)$. Since $\Phi$ is a minimal $\tau$-cusco it follows from Proposition 2.3 that there exists a non-empty open subset $U$ of $V$ such that

$$
\Phi(U) \subseteq\left(C_{m}+\frac{\varepsilon}{4} B(X)\right) \backslash\left(K+\frac{\varepsilon}{4} B(X)\right) \subseteq S\left(C_{m}, f, \delta\right)+\frac{\varepsilon}{4} B(X),
$$

so $\Phi(U)$ has diameter less than $\varepsilon$.

(ii) Given $\varepsilon>0$, consider $O_{\varepsilon} \equiv \bigcup\{$ open sets $U$ in $A: \operatorname{diam} \Phi(U)<\varepsilon\}$. Now $O_{\varepsilon}$ is open; we show that $O_{e}$ is also dense in $A$. Consider a non-empty open set $W$ in $A$. There exists $t \in W$ where $\Phi$ is $\omega$ upper semi-continuous. Therefore there exists a non-empty open subset $V$ of $W$ such that $\omega(\Phi(V))<\varepsilon / 4$. From (i) there exists a non-empty open subset $U$ of $V$ such that $\operatorname{diam} \Phi(U)<\varepsilon$. So $U \subseteq O_{\varepsilon}$ and $O_{\varepsilon}$ is dense in $A$. We conclude that $\Phi$ is single-valued and norm upper semi-continuous on $\bigcap_{1}^{\infty} O_{1 / n}$, a dense $G_{\delta}$ subset of $A$.

Theorem 2.4(i) enables us to give the counter-example for Theorem 2.1 which we promised. We give an example of a weak cusco which is not $\boldsymbol{\omega}$ upper semi-continuous. Consider an infinite dimensional Banach space $X$ and the identity mapping id from $X$ with its weak topology into $X$ with its norm topology. Now id is a minimal weak cusco on $X$. Suppose that id is $\omega$ upper semi-continuous at $x \in X$. Then given 
$\varepsilon>0$ there exists a weak open neighbourhood $V$ of $x$ such that $\omega($ id $(V))<\varepsilon / 4$. But then by Theorem 2.4(i) there exists a non-empty weak open subset $U$ of $V$ such that $\operatorname{diamid}(U)<\varepsilon$. But as a weakly open set in an infinite dimensional space $X, U=$ id $(U)$ cannot be bounded. So we conclude that id cannot be $\omega$ upper semi-continuous at any point of $X$.

A special case of Theorem 2.4(i) was given by Namioka, [12, p.527], using different methods. He showed that any non-empty weak* compact set $K$ in the dual $X^{*}$ of a Banach space $X$ with $\omega(K)<\varepsilon / 4$ has a non-empty relatively open subset $U$ with $\operatorname{diam} U<\varepsilon$. This follows from Theorem 2.4(i) by considering $\Phi$ to be the identity mapping from $K$ with the relative weak* topology, which is a Baire space, into $K$ with the relative weak* topology.

Theorem 2.4(ii) has implications for the differentiability of convex functions. A continuous convex function $\phi$ on an open convex subset $A$ of a Banach space $X$ is said to be Fréchet differentiable at $x \in A$ if $\lim _{t \rightarrow 0}(\phi(x+t y)-\phi(x)) / t$ exists and is approached uniformly for all $y \in S(X)$. A subgradient of $\phi$ at $x_{0} \in A$ is a continuous linear functional $f$ on $X$ such that $f\left(x-x_{0}\right) \leqslant \phi(x)-\phi\left(x_{0}\right)$ for all $x \in A$. The subdifferential of $\phi$ at $x_{0}$ is denoted by $\partial \phi\left(x_{0}\right)$ and is the set of subgradients of $\phi$ at $x_{0}$. The subdifferential mapping $x \rightarrow \partial \phi(x)$ is a minimal weak cusco from $A$ into subsets of $X^{*},[14, \mathrm{p} .100]$. Further, $\phi$ is Fréchet differentiable at $x \in A$ if and only if the subdifferential mapping $x \rightarrow \partial \phi(x)$ is single-valued and norm upper semicontinuous at $x,[14$, p.18]. So from Theorem 2.4 (ii) we have a result which improves [7, Corollary 2.7].

CoROLlary 2.5. A continuous convex function $\phi$ on an open convex subset $A$ of a Banach space $X$ whose subdifferential mapping $x \rightarrow \partial \phi(x)$ is $\omega$ upper semicontinuous on a dense subset of $A$ is Fréchet differentiable on a dense $G_{\delta}$ subset of A.

Theorem 2.4(ii) also reveals the special differentiability properties of reflexive Banach spaces. To examine this in full generality we need the following property of minimal weak* cuscos, [9, Lemma 3.4(ii)].

Lemma 2.6. Given a minimal weak* cusco $\Phi$ from a Baire space $A$ into subsets of the dual $X^{*}$ of a Banach space $X$, there exists a dense $G_{\delta}$ subset $D$ of $A$ such that at each $t \in D$, the real valued mapping defined on $A$ by

$$
\rho(t)=\inf \{\|f\|: f \in \Phi(t)\}
$$

is continuous and $\Phi(t)$ lies in the face of a sphere of $X^{*}$ of radius $\rho(t)$.

It follows from this lemma that a minimal weak* cusco $\Phi$ from a Baire space $A$ into subsets of the dual $X^{*}$ of a Banach space $X$ is locally bounded on a dense $G_{\delta}$ subset $D$ of $A$. So we have the following well known result. 
Corollary 2.7. Every minimal weak* cusco $\Phi$ from a Baire space $A$ into subsets of the dual $X^{*}$ of a reflexive Banach space is single-valued and norm upper semi-continuous on a dense $G_{\delta}$ subset $D$ of $A$. In particular, a reflexive Banach space is an Asplund space.

Part of the study of Asplund spaces has been to find conditions sufficient for a Banach space to be Asplund. Many of these conditions have concerned differentiability properties of the norm. Given a Banach space $X$, for each $x \in S(X)$ we denote by $D(x)$ the set $\left\{f \in S\left(X^{*}\right): f(x)=1\right\}$. The set-valued mapping $x \rightarrow D(x)$ from $S(X)$ into subsets of $S\left(X^{*}\right)$ is called the duality mapping on $S(X)$. It is known that a Banach space $X$ whose duality mapping $x \rightarrow D(x)$ is a weak cusco on $S(X)$ is an Asplund space, $[5$, p.106]. So we can make the following deduction from Theorem 2.1(ii).

Theorem 2.8. A Banach space $X$ with duality mapping $x \rightarrow D(x)$ w upper semi-continuous on $S(X)$ is an Asplund space.

\section{Denting Point Structure For ClOSED bounded CONVEX SETS}

We now examine the implications of Theorem 2.4 for the structure of closed bounded convex sets in a Banach space.

For a closed bounded convex set $K$ with $0 \in K$ we define the polar of $K$ as the set $K^{0} \equiv\left\{f \in X^{*}: f(x) \leqslant 1\right.$ for all $\left.x \in K\right\}$. If $0 \in$ int $K$ then $K^{0}$ is weak* compact and convex. We denote by $K^{00}$ the polar of $K^{0}$ in $X^{* *}$. Since $K$ is bounded then $0 \in$ int $K^{0}$ and so $K^{00}$ is weak* compact and convex.

We need the following basic properties of slices and polars, (see [7, Lemma 3.1]). We use ${ }^{\wedge}$ to denote natural embedding elements.

Proposition 3.1. Consider a closed bounded convex set $K$ with $0 \in K$ in a Banach space $X$. Then

(i) $\widehat{K}$ is weak ${ }^{*}$ dense in $K^{00}$, and

(ii) given $f \in K^{*} \backslash\{0\}$ and $0<r<\sup f(K)=\sup \hat{f}\left(K^{00}\right), S(\widehat{K}, \widehat{f}, r)$ is weak* dense in $S\left(K^{00}, \widehat{f}, r\right)$.

Consider a non-empty closed bounded convex set $K$ in a Banach space $X$. We say that $x \in \partial K$ is a denting point, ( $\alpha$ denting point, $\omega$ denting point) of $K$ if given $\varepsilon>0$, $x$ is contained in a slice of $K$ of diameter ( $\alpha$ index, $\omega$ index) less than $\varepsilon$. It is clear that if $x$ is a denting point of $K$ then $x$ is an $\alpha$ denting point of $K$ and if $x$ is an $\alpha$ denting point of $K$ then $x$ is an $\omega$ denting point of $K$, but generally the implications are not reversed. Similarly, for a non-empty closed bounded convex set $K$ in the dual of a Banach space $X$, we say that $f \in \partial K$ is a weak* denting point, (weak $\alpha$ denting point, weak ${ }^{*} \omega$ denting point) of $K$ if given $\varepsilon>0, f$ is contained in a weak* slice of $K$ 
of diameter ( $\alpha$ index, $\omega$ index) less than $\varepsilon$. For a non-empty closed bounded set $K$ in a Banach space $X$, it is well known that every denting point of $K$ is an extreme point of $K$. But since every finite dimensional Banach space $X_{n}$ has every point of $S\left(X_{n}\right)$ an $\alpha$ denting point of $B\left(X_{n}\right), \alpha$ denting points of $K$ and by implication $\omega$ denting points of $K$, are not necessarily extreme points of $K$.

We need the following relations between the various types of denting points of a closed bounded convex set and those of its double polar.

Lemma 3.2. Consider a closed bounded convex set $K$ with $0 \in K$ in a Banach space $X$.

(i) The set of weak* denting points of $K^{00}$ is contained in $\widehat{K}$.

(ii) Given $f \in X^{*} \backslash\{0\}$ and $0<r<\sup f(K)=\sup \widehat{f}\left(K^{00}\right)$, $\operatorname{diam} S(K, f, r)=\operatorname{diam} S\left(K^{00}, \hat{f}, r\right), \alpha(S(K, f, r))=\alpha\left(S\left(K^{00}, \hat{f}, r\right)\right)$, and $\omega(S(K, f, r)) \geqslant \omega\left(S\left(K^{00}, \hat{f}, r\right)\right)$.

(iii) $\widehat{x}$ is a weak ${ }^{*}$ denting point of $K^{00}$ if and only if $x$ is a denting point of $K, \widehat{x}$ is a weak ${ }^{*} \alpha$ denting (and extreme) point of $K^{00}$ if $x$ is an $\alpha$ denting (and extreme) point of $K, \widehat{x}$ is a weak ${ }^{*} \omega$ denting (and extreme) point of $K^{00}$ if $x$ is an $\omega$ denting (and extreme) point of $K$.

Proof: (i) Consider $F \in X^{* *} \backslash \hat{X}$. Then $d(F, \hat{X}) \equiv d>0$ and $B(F, d / 2)$ contains no points of $\widehat{X}$. But by Proposition 3.1(ii) every weak* slice of $K^{00}$ contains points of $\widehat{K}$ so $F$ is not contained in any weak* slice of $K^{00}$ of diameter less than $d / 2$ and so is not a weak* denting point of $K$.

(ii) Using the fact that for any bounded set $E$ in $X, \operatorname{diam} \overline{\mathrm{CO}}^{\omega^{*}} \widehat{E}=\operatorname{diam} E$, we can establish the equalities for diamter and $\alpha$ index. Suppose that $\omega(S(K, f, r))=\varepsilon$. Then given $\varepsilon^{\prime}>\varepsilon$, there exists a weakly compact set $C$ in $X$ such that $S(K, f, r) \subseteq$ $C+\varepsilon^{\prime} B(X)$ so $S(\widehat{K}, \widehat{f}, r) \subseteq \widehat{C}+\varepsilon^{\prime} B\left(X^{* *}\right)$ which is weak* closed. By Proposition 3.1(ii), $S\left(K^{00}, \widehat{f}, r\right) \subseteq \widehat{C}+\varepsilon^{\prime} B\left(X^{* *}\right)$. Since $\widehat{C}$ is a weakly compact set in $X^{* *}$ we conclude that $\omega\left(S\left(K^{00}, \hat{f}, r\right)\right) \leqslant \varepsilon$.

(iii) If for $x \in K$, given $\varepsilon>0$ there exists $f \in X^{*} \backslash\{0\}$ and $\delta>0$ such that $x \in$ $S(K, f, \delta)$ and $\operatorname{diam} S(K, f, \delta)<\varepsilon,(\alpha(S(K, f, \delta))<\varepsilon, \omega(S(K, f, \delta))<\varepsilon)$, then $\widehat{x} \in$ $S\left(K^{00}, \widehat{f}, \delta\right)$ and from (ii) our primary result follows. If for $x \in K$, given $\varepsilon>0$ there exists $f \in X^{*} \backslash\{0\}$ and $\delta>0$ such that $\widehat{x} \in S\left(K^{00}, \widehat{f}, \delta\right)$ and $\operatorname{diam} S\left(K^{00}, \widehat{f}, \delta\right)<\varepsilon$, from (i) we have that $x$ is a denting point of $K$.

For the secondary result it is sufficient to show that if $x$ is an $\omega$ denting extreme point of $K$ then $\widehat{x}$ is an extreme point of $K^{00}$. Suppose that there exists $F$ and 
$G \in K^{00}, F \neq G$ such that $\widehat{x}=\lambda F+(1-\lambda) G$ for some $0<\lambda<1$. Since $x$ is an extreme point of $K$ which is closed and convex and $\widehat{K}$ is weak dense in $K^{00}$ then $F$ and $G \in X^{* *} \backslash \widehat{X}$. For $d \equiv \min \{d(F, \widehat{X}), d(G, \widehat{X})\}>0$ consider $f \in X^{*} \backslash\{0\}$ and $\delta>0$ such that $x \in S(K, f, \delta)$ and $\omega(S(K, f, \delta))<d / 2$. Then there exists a weakly compact set $C$ in $X$ such that $S(K, f, \delta) \subseteq C+(d / 2) B(X)$. But $S(\widehat{K}, \hat{f}, \delta)$ is weak* dense in $S\left(K^{00}, \widehat{f}, \delta\right)$ so $S\left(K^{00}, \widehat{f}, \delta\right) \subseteq \widehat{C}+(d / 2) B\left(X^{* *}\right)$. Now at least one of $F$ and $G$ is in $S\left(K^{00}, \widehat{f}, \delta\right)$. Suppose $F \in S\left(K^{00}, \widehat{f}, \delta\right)$. Then $F \in \widehat{C}+(d / 2) B\left(X^{* *}\right)$ which implies that $d(F, \widehat{X}) \leqslant d / 2$ contradicting $d(F, \widehat{X}) \geqslant d$. We conclude that $\widehat{x}$ is an extreme point of $K^{00}$.

Given a closed bounded convex set $K$ in a Banach space $X$ we say that $K$ has property $\alpha$ for $f \in X^{*} \backslash\{0\}$ if given $\varepsilon>0$ there exists $\delta(\varepsilon, f)>0$ such that $\alpha(S(K, f, \delta))<\varepsilon$. Similarly, we say that $K$ has property $\omega$ for $f \in X^{*} \backslash\{0\}$ if given $\varepsilon>0$ there exists $\delta(\varepsilon, f)>0$ such that $\omega(S(K, f, \delta))<\varepsilon$. Both properties have been studied in relation to drop properties of $K . K$ is said to have the drop property (weak drop property) if for every closed (weakly sequentially closed) set $C$ disjoint from $K$ there exists $x \in C$ such that $C \cap \operatorname{co}\{x, K\}=\{x\}$. Kutzarova showed, $[10$, p.284] that $K$ with int $K \neq \emptyset$ has the drop property if and only if it has property $\alpha$ for each $f \in X^{*} \backslash\{0\}$ and Kutzarova and Papini showed, [11, Propositions 1 and 5] that $K$ with int $K \neq \emptyset$ has the weak drop property if and only if it has property $\omega$ for each $f \in X^{*} \backslash\{0\}$.

For a closed bounded convex set $K$ with $0 \in \operatorname{int} K$, the gauge $p$ of $K$ defined by $p(x)=\inf \{\lambda>0: x \in \lambda K\}$ is a continuous sublinear functional on $X$. For a closed bounded convex set $K$ with $0 \in$ int $K$, there is a very satisfying duality between $\alpha$ upper semi-continuity of the subdifferential mapping $x \rightarrow \partial p(x)$ for $p$ the gauge of $K$ and property $\alpha$ for $K^{0}$ and between property $\alpha$ for $K$ and $\alpha$ upper semi-continuity of the subdifferential mapping $f \rightarrow \partial p(f)$ for $p$ the gauge of $K^{0},[7$, Theorem 3.2]. We now explore similar relations for $\omega$ upper semi-continuity and property $\omega$. We need the following basic properties relating slices of $K^{0}$ to the subdifferential mapping $x \rightarrow \partial p(x)$, (see [7, Theorem 3.2(i)]).

Proposition 3.3. Consider a closed bounded convex set $K$ with $0 \in$ int $K$ in a Banach space $X$ with $p$ the gauge of $K$.

(i) Given $x \in X \backslash\{0\}$ and $0<\delta^{2}<\sup \widehat{x}\left(K^{0}\right)$,

$$
\overline{S\left(K^{0}, \widehat{x}, \delta^{2}\right)} \subseteq \partial p(B(x ; \delta))+\delta B\left(X^{*}\right) \text {. }
$$

(ii) Given $x \in X \backslash\{0\}$ and $0<r<\sup \widehat{x}\left(K^{0}\right)$ there exists $\delta>0$ such that

$$
\partial p(B(x ; \delta)) \subseteq S\left(K^{0}, \widehat{x}, r\right) .
$$


Lemma 3.4. Consider a closed bounded convex set $K$ with $0 \in$ int $K$ in a Banach space $X$.

(i) For $p$ the gauge of $K$ on $X$, the subdifferential mapping $x \rightarrow \partial p(x)$ is $\omega$ upper semi-continuous at $x \in X \backslash\{0\}$ if and only if $K^{0}$ has property $\omega$ for $\widehat{x}$.

(ii) For $p$ the gauge of $K^{0}$ on $X^{*}$, the subdifferential mapping $f \rightarrow \partial p(f)$ is $\omega$ upper-semi-continuous at $f \in X^{*} \backslash\{0\}$ if $K$ has property $\omega$ for $f$.

Proof: (i) If $x \rightarrow \partial p(x)$ is $\omega$ upper semi-continuous at $x$, given $\varepsilon>0$ there exists a weakly compact set $C$ in $X^{*}$ and $0<\delta<\varepsilon$ such that $\partial p(B(x ; \delta)) \subseteq C+\varepsilon B\left(X^{*}\right)$. Then by Proposition 3.3(i), $S\left(K^{0}, \widehat{x}, \delta^{2}\right) \subseteq C+2 \varepsilon B\left(X^{*}\right)$; that is, $K^{0}$ has property $\omega$ for $x$.

Conversely, if $K^{0}$ has property $\omega$ for $\widehat{x}$, given $\varepsilon>0$ there exists a weakly compact set $C$ in $X^{*}$ and $0<r<\sup \widehat{x}\left(K^{0}\right)$ such that $S\left(K^{0}, \widehat{x}, r\right) \subseteq C+\varepsilon B\left(X^{*}\right)$. Then by Proposition 3.3(ii) there exists a $\delta>0$ such that $\partial p(B(x ; \delta)) \subseteq C+\varepsilon B\left(X^{*}\right)$; that is, $x \rightarrow \partial p(x)$ is $\omega$ upper semi-continuous at $x$.

(ii) If $K$ has property $\omega$ for $f$, given $\varepsilon>0$ there exists a weakly compact set $C$ in $X$ and $0<r<\sup f(K)=\sup \widehat{f}\left(K^{00}\right)$ such that $S(K, f, r) \subseteq C+\varepsilon B\left(X^{*}\right)$. Then $S(\widehat{K}, \widehat{f}, r) \subseteq \widehat{C}+\varepsilon B\left(X^{* *}\right)$ and $\widehat{C}$ is weakly compact in $X^{* *}$ and $\widehat{C}+\varepsilon B\left(X^{* *}\right)$ is weak* closed. By Proposition 3.1(ii), $S\left(K^{00}, \widehat{f}, r\right) \subseteq \widehat{C}+\varepsilon B\left(X^{* *}\right)$; that is $K^{00}$ has property $\omega$ for $f$. Then by (i), $f \rightarrow \partial p(f)$ is $\omega$ upper semi-continuous at $f$.

It is not known whether the converse to Lemma 3.4(ii) holds locally. The converse depends on knowing whether given $f \in X^{*} \backslash\{0\}, K^{00}$ having property $\omega$ for $f$ implies that $K$ has property $\omega$ for $f$. However, the converse does hold globally because if the subdifferential mapping $f \rightarrow \partial p(f)$ for $p$ the gauge of $K^{0}$ is $\omega$ upper semi-continuous on $X^{*} \backslash\{0\}$ then by Theorem 2.1(ii), $f \rightarrow \partial p(f)$ is a weak cusco on $X^{*} \backslash\{0\}$ and this implies that $K$ is weakly compact, $[6, p .381]$. Since int $K \neq \emptyset$. we have that $X$ is reflexive and in this case $K$ has property $\omega$ for all $f \in X^{*} \backslash\{0\}$.

LEMMA 3.5 .

(i) Consider a non-empty weak* compact convex set $K$ in the dual $X^{*}$ of a Banach space $X$. Any weak* slice of $K$ which contains a weak* $\omega$ denting extreme point of $K$ contains the closure of a weak* slice of arbitrarily small diameter.

(ii) Consider a non-empty closed bounded convex set $K$ in a Banach space $X$. Any slice of $K$ which contains an $\omega$ denting extreme point of $K$ contains the closure of a slice of arbitrarily small diameter.

Proof: We may assume in both cases that $0 \in K$. 
(i) Consider $x \in X \backslash\{0\}$ and $\delta>0$ and $S(K, \widehat{x}, \delta)$ containing a weak* $\omega$ extreme denting point $f$ of $K$. Then given $\varepsilon>0$ there exists $y \in X \backslash\{0\}$ and $\delta^{\prime}>0$ and such that $f \in S(K, \widehat{x}, \delta) \cap S\left(K, \widehat{y}, \delta^{\prime}\right)$ and $\omega\left(S\left(K, \widehat{y}, \delta^{\prime}\right)\right)<\varepsilon / 8$. Now there exists $0<\lambda<1$ such that $f \in S(K, \widehat{x}, \lambda \delta) \cap S\left(K, \widehat{y}, \delta^{\prime}\right)$. Since $f$ is an extreme point $K$, $f \notin \overline{\mathrm{co}}^{w^{*}}\left(K \backslash\left(S(K, \widehat{x}, \lambda \delta) \cap S\left(K, \widehat{y}, \delta^{\prime}\right)\right)\right)$. So there exists $z \in X \backslash\{0\}$ and $\delta^{\prime \prime}>0$ such that $f \in S\left(K, \widehat{z}, \delta^{\prime \prime}\right)$ and $S\left(K, \widehat{z}, \delta^{\prime \prime}\right) \cap \overline{\operatorname{co}}^{\omega^{*}}\left(K \backslash\left(S(K, \widehat{x}, \lambda \delta) \cap S\left(K, \widehat{y}, \delta^{\prime}\right)\right)\right)=\emptyset$. Define the functional $p$ on $X$ by $p(x)=\sup \{f(x): f \in K\}$. Then $p$ is a continuous sublinear functional on $X$. Also $p$ is the gauge of $C \equiv\{x \in X: p(x)<1\}$ and $K=C^{0}$. Now the set of weak* support points of $K$ is dense in $\partial K,[15$, p.180]. Consider $\widehat{w} \in \widehat{X}$ a weak* support functional of $K$ where $\partial p(w) \cap S\left(K, \widehat{z}, \delta^{\prime \prime}\right) \neq \emptyset$. By Proposition 2.3 there exists a non-empty open subset $V$ of a neighbourhood of $w$ such that $\partial p(V) \subseteq S\left(K, \widehat{z}, \delta^{\prime \prime}\right)$. Then $\omega(\partial p(V))<\varepsilon / 8$ and from Theorem 2.4(i) there exists a non-empty open subset $U$ of $V$ such that $\operatorname{diam} \partial p(U)<\varepsilon / 2$. Choose $x_{0} \in U \backslash\{0\}$ and $0<\delta_{1}<\min (\varepsilon / 4, \lambda \delta,(1-\lambda) \delta)$ such that $B\left(x_{0} ; \delta_{1}\right) \subseteq U$. Then from Proposition 3.3(i), $\overline{S\left(K, \widehat{x}_{0}, \delta_{1}^{2}\right)} \subseteq \partial p\left(B\left(x_{0} ; \delta_{1}\right)\right)+\delta_{1} B\left(X^{*}\right)$, so diam $S\left(K, \widehat{x}_{0}, \delta_{1}^{2}\right)<\varepsilon$. Notice that $\left(\partial p\left(B\left(x_{0} ; \delta_{1}\right)\right)+\delta_{1} B\left(X^{*}\right)\right) \cap K \subseteq\left(S(K, \widehat{x}, \lambda \delta)+(1-\lambda) \delta B\left(X^{*}\right)\right) \cap K \subseteq S(K, \widehat{x}, \delta)$.

(ii) Consider $f \in X^{*} \backslash\{0\}$ and $\delta>0$ where $S(K, f, \delta)$ contains an $\omega$ denting extreme point $x$ of $K$. Then $\widehat{x} \in S\left(K^{00}, \widehat{f}, \delta\right)$ and by Lemma 3.2(ii), $\widehat{x}$ is a weak* $\omega$ denting extreme point of $K^{00}$. So by (i) given $\varepsilon>0$ there exists $g \in X^{*} \backslash\{0\}$ and $\delta^{\prime}>0$ such that $\overline{S\left(K^{00}, \widehat{g}, \delta^{\prime}\right)} \subseteq S\left(K^{00}, \widehat{f}, \delta\right)$ and $\operatorname{diam} S\left(K^{00}, \widehat{g}, \delta^{\prime}\right)<\varepsilon$. Then by Proposition 3.1(ii), $\emptyset \neq S\left(K, g, \delta^{\prime}\right) \subseteq S(K, f, \delta)$ and $\operatorname{diam} S\left(K, g, \delta^{\prime}\right)<\varepsilon$.

It is interesting to note that in any Banach space there is an equivalence of denting point structure for a closed bounded convex set. We begin with the equivalence theorem for such sets in the dual and then use it to establish the corresponding theorem for sets in any Banach space.

THEOREM 3.6. Consider a non-empty weak* compact subset $K$ of the dual of a Banach space $X$. Then the following are equivalent.

(i) $K$ is the weak* closed convex hull of its weak* denting points.

(ii) $K$ is the weak $k^{*}$ closed convex hull of its weak ${ }^{*} \alpha$ denting extreme points.

(iii) $K$ is the weak ${ }^{*}$ closed convex hull of its weak* $\omega$ denting extreme points.

PROOF: Since every weak* denting point is a weak* $\alpha$ denting point and every weak* $\alpha$ denting point is a weak* $\omega$ denting point, the implications (i) $\Rightarrow$ (ii) $\Rightarrow$ (iii) are obvious. It remains to prove (iii) $\Rightarrow$ (i).

Suppose that $K$ is the weak* closed convex hull of its weak* $\omega$ denting extreme points but not of its weak* denting points. Denote by $M$ the weak* closed convex hull of the weak ${ }^{*}$ denting points of $K$. There exists $x \in X \backslash\{0\}$ and $\delta>0$ such that $S(K, \widehat{x}, \delta) \cap M=\emptyset$ and there exists a weak* $\omega$ denting extreme point 
$f \in S(K, \widehat{x}, \delta)$. By Lemma 3.5(i) there exists $x_{1} \in X \backslash\{0\}$ and $\delta_{1}>0$ such that $\overline{S\left(K, \widehat{x}_{1}, \delta_{1}\right)} \subseteq S(K, \widehat{x}, \delta)$ and $\operatorname{diam} S\left(K, \widehat{x}_{1}, \delta_{1}\right)<1 / 2$. Again there exists a weak $\omega$ denting extreme point $f_{1} \in S\left(K, \widehat{x}_{1}, \delta_{1}\right)$ and by Lemma 3.5(i) there exists $x_{2} \in X \backslash\{0\}$ and $\delta_{2}>0$ such that $\overline{S\left(K, \widehat{x}_{2}, \delta_{2}\right)} \subseteq S\left(K, \widehat{x}_{1}, \delta_{1}\right)$ and $\operatorname{diam} S\left(K, \widehat{x}_{2}, \delta_{2}\right)<1 / 4$. Continuing, we construct a nested sequence of weak* slices $\left\{S\left(K, \widehat{x}_{n}, \delta_{n}\right)\right\}$ such that $\operatorname{diam} S\left(K, \widehat{x}_{n}, \delta_{n}\right)<1 / 2^{n}$. Now $\bigcap_{1}^{\infty} S\left(K, \widehat{x}_{n}, \delta_{n}\right)=\bigcap_{1}^{\infty} \overline{S\left(K, \widehat{x}_{n}, \delta_{n}\right)}$ is a singleton and this point is clearly a weak* denting point of $K$. But as this point lies in $K \backslash M$ we have contradicted our supposition about $M$.

The dual theorem follows by Lemma 3.5(ii) using the same argument.

THEOREM 3.7. Consider a closed bounded convex subset $K$ of a Banach space $X$. The following are equivalent.

(i) $K$ is the closed convex hull of its denting points.

(ii) $K$ is the closed convex hull of its $\alpha$ denting extreme points.

(iii) $K$ is the closed convex hull of its $\omega$ denting extreme points.

\section{IMPLICATIONS FOR THE DIFFERENTIABILITY OF CONVEX FUNCTIONS}

The classical characterisation theorem for Asplund spaces given by Namioka and Phelps [13, p.739], and generalised in [7, Theorems 4.9 and 4.10] for the $\alpha$ index, has comparable form for the $\omega$ index. The theorem is expressed in terms of exposed point structure.

Consider a closed bounded convex set $K$ in a Banach space $X$. We say that $x \in K$ is a strongly exposed point of $K$ if there exists $f \in S\left(X^{*}\right)$ such that for every $\varepsilon>0$ there exists $\delta>0$ such that $x \in S(K, f, \delta)$ and $\operatorname{diam} S(K, f, \delta)<\varepsilon$; we say that $f$ strongly exposes $K$ at $x$. Generalising, we say that a subset $E$ of $K$ is $\alpha$ strongly exposed, ( $\omega$ strongly exposed) if there exists $f \in S\left(X^{*}\right)$ such that $E=\bigcap_{\delta \rightarrow 0} S(K, f, \delta)$ and $K$ has property $\alpha$ for $f$, (property $\omega$ for $f$ ); we say that $f$ a strongly exposes ( $\omega$ strongly exposes) $K$ at $E$. For a closed bounded convex set $K$ in the dual $X^{*}$ we say that $f \in K$ is a weak* strongly exposed point of $K$ if there exists $\widehat{x} \in S(\widehat{X})$ which strongly exposes $K$ at $f$ and a subset $E$ of $K$ is weak $\alpha$ strongly exposed, (weak $\omega$ strongly exposed) if there exists $\widehat{x} \in S(\widehat{X})$ which $\alpha$ strongly exposes, ( $\omega$ strongly exposes) $K$ at $E$.

We note that a singleton subset of $K$ is $\alpha$ strongly exposed if and only if it is a strongly exposed point of $K,[7$, Proposition 4.8]. A comparable result does not hold for singleton subsets which are $\omega$ strongly exposed. A reflexive Banach space with a rotund but not locally uniformly rotund norm has every singleton subset of the unit sphere $\omega$ strongly exposed but not all are strongly exposed. 
For a closed bounded convex set $K$ in the dual $X^{*}$, a subset $E$ of $K$ which is weak* $\omega$ strongly exposed is weak* compact and so by the Krein-Milman Theorem contains an extreme point which is an extreme point of $K$. So $E$ always contains a weak $^{*} \omega$ denting extreme point of $K$. Then the proof of the following characterisation theorem is a direct consequence of Theorem 3.6.

Theorem 4.1. For a Banach space $X$ the following are equivalent;

(i) every continuous convex function $\phi$ on an open convex subset $A$ of $X$ is Fréchet differentiable on a dense $G_{6}$ subset of $A$,

(ii) every weak* compact convex subset of $X^{*}$ is the weak* closed convex hull of its weak* strongly exposed points,

(iii) every weak* compact convex subset of $X^{*}$ is the weak* closed convex hull of its weak* $\omega$ strongly exposed points.

For a closed bounded convex set $K$ in a Banach space $X$, it follows from the $\omega$ index property (v) that a subset $E$ of $K$ which is $\omega$ strongly exposed is weakly compact and again by the Krein-Milman Theorem contains an $\omega$ denting extreme point of $K$. So the proof of the following dual characterisation theorem is a direct consequence of Theorem 3.7.

THEOREM 4.2. For a Banach space $X$ the following are equivalent;

(i) every continuous weak* lower semi-continuous convex function $\phi$ on an open convex subset $A$ of $X^{*}$ is Fréchet differentiable on a dense $G_{\delta}$ subset of $A$,

(ii) every closed bounded convex subset of $X$ is the closed convex hull of its strongly exposed points,

(iii) every closed bounded convex subset of $X$ is the closed convex hull of its $\omega$ strongly exposed points.

There are separable Banach spaces wl.ich do not have the differentiability properties of Theorem 4.2. But it has recently been shown, [9, Theorem 3.5], that there is a large class of Banach spaces, including the separable spaces, where every continuous convex function on an open convex subset of the dual is Fréchet differentiable on a dense $G_{\delta}$ subset of its domain provided that the set of points where the function has a weak* continuous subgradient is residual in its domain. Spaces of this class are those which can be equivalently renormed to have every point of the unit sphere a denting point of the closed unit ball. This result has been extended, [7, Theorem 4.5], to include spaces which can be equivalently renormed to have every point of the unit sphere an $\alpha$ denting point of the closed unit ball. The result can be extended further using the weak index of non-compactness.

THEOREM 4.3. Consider a Banach space $X$ which can be equivalently renormed 
to have every point of $S(X)$ an $\omega$ denting point of $B(X)$. Then every minimal weak* cusco $\Phi$ from a Baire space $A$ into subsets of $X^{* *}$ for which the set $G \equiv\{t \in A: \Phi(t) \cap$ $\widehat{X} \neq \emptyset\}$ is residual in $A$, is single-valued and norm upper semi-continuous on a dense $G_{\delta}$ subset of $A$. In particular, every continuous convex function $\phi$ on an open convex set $A$ in $X^{*}$ for which the set $G \equiv\{f \in A: \partial \phi(f) \cap \widehat{X} \neq \emptyset\}$ is residual in $A$, is Fréchet differentiable on a dense $G_{\delta}$ subset of $A$.

Proof: Consider $X$ so renormed. Given $\varepsilon>0$, consider $0_{\varepsilon} \equiv \cup$ \{open sets $V$ in $A: \omega(\Phi(V))<\varepsilon\}$. Now $0_{\varepsilon}$ is open; we show that $0_{\varepsilon}$ is dense in $A$. From Lemma 2.6 there exists a dense $G_{\delta}$ subset $G_{1}$ of $A$ such that at every point $t \in G_{1}$ the mapping $\rho$ where $\rho(t)=\inf \{\|f\|: f \in \Phi(t)\}$, is continuous and $\Phi(t)$ lies in the face of a sphere of $X^{* *}$ of radius $\rho(t)$. Now $G \cap G_{1}$ is residual in $A$. Consider a non-empty open subset $W$ of $A$ and $t_{0} \in G \cap G_{1} \cap W$. There exists some $\widehat{x}_{0} \in \Phi\left(t_{0}\right) \cap \widehat{X}$. If $x_{0}=0$, then since $\rho$ is continuous at $t_{0}$ there exists an open neighbourhood $U$ of $t_{0}$ such that $\Phi(t) \cap \varepsilon B\left(X^{* *}\right) \neq$ $\emptyset$ for all $t \in U$. Then by Proposition 2.3, $\Phi(U) \subseteq \varepsilon B\left(X^{* *}\right)$ so $U \subseteq O_{\epsilon} \cap W$. If $x_{0} \neq 0$, then $x_{0}$ is an $\omega$ denting point of $\rho\left(t_{0}\right) B(X)$ so by Lemma 3.2(iii), $\widehat{x}_{0}$ is a weak* $\omega$ denting point of $\rho\left(t_{0}\right) B\left(X^{* *}\right)$. Then there exists $g \in S\left(X^{*}\right)$ and $\delta>0$ such that $\widehat{x}_{0} \in S\left(\rho\left(t_{0}\right) B\left(X^{* *}\right), \widehat{g}, \delta\right)$ and $\omega\left(S\left(\rho\left(t_{0}\right)\right) B\left(X^{* *}\right), \widehat{g}, \delta\right)<\varepsilon / 2$. We can choose $1<\lambda<2$ such that $\widehat{x}_{0} \in S\left(\lambda \rho\left(t_{0}\right) B\left(X^{* *}\right), \widehat{g}, \lambda \delta\right)=\lambda S\left(\rho\left(t_{0}\right) B\left(X^{* *}\right), \widehat{g}, \delta\right)$ and then by the $\omega$ index property (iv), $\omega\left(\lambda S\left(\rho\left(t_{0}\right)\right) B\left(X^{* *}\right), \widehat{g}, \delta\right)<\varepsilon$. Since $\rho$ is continuous at $t_{0}$ there exists an open subset $V^{\prime}$ of $W$ containing $t_{0}$ such that $\Phi(t) \cap \lambda \rho\left(t_{0}\right) B\left(X^{* *}\right) \neq \emptyset$ for all $t \in V^{\prime}$. So by Proposition 2.3, $\Phi\left(V^{\prime}\right) \subseteq \lambda \rho\left(t_{0}\right) B\left(X^{* *}\right)$. Since $\Phi\left(V^{\prime}\right) \nsubseteq\{F \in$ $\left.X^{* *}: F(g) \leqslant \lambda \rho\left(t_{0}\right)-\lambda \delta\right\}$ then again by Proposition 2.3, there exists a non-empty open subset $V$ of $V^{\prime}$ and so of $W$ such that $\Phi(V) \subseteq S\left(\lambda \rho\left(t_{0}\right) B\left(X^{* *}\right), \widehat{g}, \lambda \delta\right)$ and so $\omega(\Phi(V))<\varepsilon$. We conclude that $\Phi$ is $\omega$ upper semi-continuous on the dense $G_{\delta}$ subset $\bigcap_{1}^{\infty} O_{1 / n}$ of $A$ and our result follows from Theorem 2.4(ii).

We noted previously that the subdifferential mapping $f \rightarrow \partial \phi(f)$ of a continuous convex function $\phi$ on an open convex subset $A$ of $X^{*}$ is a minimal weak* cusco from $A$ into subsets of $X^{* *}$, so from Corollary $2.5, \phi$ is Fréchet differentiable on a dense $G_{\delta}$ subset of $A$.

The theorem as it now stands includes the earlier ones with spheres consisting of denting points and spheres consisting of $\alpha$ denting points. Troyanski [16, p.306; 18, Theorem 2.3] has shown that spaces which have every point of the unit sphere a denting point ( $\alpha$ denting point) of the closed unit ball can be equivalently renormed to have locally uniformly rotund norm. It remains an open problem whether spaces of the class satisfying the hypothesis of our theorem can be equivalently renormed to have locally uniformly rotund norm.

As with the cases where every point of the unit sphere is a denting point or an 
$\alpha$-denting point, we have a differentiability property for the dual norm of a Banach space where every point of the unit sphere is an $\omega$-denting point. This generalises the earlier results given in $[9$, Theorem 3.2] and [7, Theorem 4.7]. We need the following elementary property for slices, [7, Lemma 4.6].

Lemma 4.4. For a Banach space $X$ and $x \in S(X)$ and any slice of $B(X)$ determined by $f \in S\left(X^{*}\right)$ and containing $x$, there exists $\varepsilon>0$ such for all $g \in S\left(X^{*}\right)$ where $\|g-f\|<\varepsilon$ there exists a slice of $B(X)$ determined by $g$ which contains $x$ and is contained in the slice determined by $f$.

Given $\varepsilon>0$, we denote by $\omega_{\varepsilon}\left(S\left(X^{*}\right)\right)$ the set of points $S\left(X^{*}\right)$ which determine slices of $B(X)$ with $\omega$ index less than $\varepsilon$. From Lemma 4.4 we see that $\omega_{e}\left(S\left(X^{*}\right)\right)$ is open in $S\left(X^{*}\right)$. Further $B(X)$ has property $\omega$ for all $f \in \bigcap_{1}^{\infty} \omega_{1 / n}\left(S\left(X^{*}\right)\right)$.

THEOREM 4.5. A Banach space $X$ where every point of $S(X)$ is an $\omega$ denting point of $B(X)$ has dual norm Fréchet differentiable on a dense $G_{\delta}$ subset of $X^{*}$.

Proof: Consider $f \in S\left(X^{*}\right)$ which attains its norm on $S(X)$ say at $x \in S(X)$. Then $x$ is an $\omega$-denting point of $B(X)$, so given $0<\varepsilon<1$ there exists $g \in S\left(X^{*}\right)$ and $0<\delta<1$ such that $x \in S(B(X), g, \delta)$ and $\omega(S(B(X), g, \delta))<\varepsilon$. For $0<\eta<\varepsilon$ consider $h=\eta g+(1-\eta) f$. Then $\|h-f\|<2 \eta$. Writing $K \equiv B(X) \backslash S(B(X), g, \delta)$, we have

$$
\sup h(K) \leqslant \eta g(K)+(1-\eta) \sup f(K)<\eta g(x)+(1-\eta) f(x)=h(x) .
$$

So $h$ separates $x$ from $K$ and defines a slice of $B(X)$ containing $x$ but contained in $S(B(X), f, \delta)$. Then $h \in \omega_{\varepsilon}\left(S\left(X^{*}\right)\right)$. From the Bishop-Phelps Theorem we conclude that $\omega_{\varepsilon}\left(S\left(X^{*}\right)\right)$ is dense in $S\left(X^{*}\right)$ and since $\omega_{\varepsilon}\left(S\left(X^{*}\right)\right)$ is open in $S\left(X^{*}\right)$, $\bigcap_{1}^{\infty} \omega_{1 / n}\left(S\left(X^{*}\right)\right)$ is a dense $G_{\delta}$ subset of $S\left(X^{*}\right)$. Then $B(X)$ has property $\omega$ for all $f \in \bigcap_{1}^{\infty} \omega_{1 / n}\left(S\left(X^{*}\right)\right)$ and from Lemma 3.4(ii), the duality mapping $f \rightarrow D(f)$ on $S\left(X^{*}\right)$ is $\omega$ upper semi-continuous on $\bigcap_{1}^{\infty} \omega_{1 / n}\left(S\left(X^{*}\right)\right)$. We deduce from Theorem 2.4(ii) that the norm of $X^{*}$ is Fréchet differentiable on a dense $G_{\delta}$ subset of $S\left(X^{*}\right)$.

In a finite dimensional Banach space $X_{n}$ every point of $S\left(X_{n}\right)$ is an $\alpha$ denting point of $B\left(X_{n}\right)$ and in a reflexive Banach space $X$ every point of $S(X)$ is an $\omega$ denting point of $B(X)$. Troyanski [17, Example 4.4] has constructed for $c_{0}$ an equivalent norm which is not rotund and where the weak and norm topologies do not agree at every point of $S\left(c_{0}\right)$ but where every point of $S\left(c_{0}\right)$ is an $\alpha$ denting point of $B\left(c_{0}\right)$. While Theorem 3.8 tells us that the closed unit ball for spaces of both classes we have considered is the closed convex hull of its denting points, nevertheless the denting points are not 
necessarily dense in the unit sphere. Theorem 4.5 does give us the further information that the closed unit ball for spaces where every point of the unit sphere is an $\alpha$ denting point or $\omega$ denting point is the closed convex hull of its strongly exposed points, [14, p.87].

\section{REFERENCES}

[1] Józef Banás and Jesus Rivero, 'On measures of weak non-compactness', Ann. Mat. Pura Appl. (4) 151 (1988), 213-224.

[2] J. Bourgain, 'Strongly exposed points in weakly compact convex sets in Banach spaces', Proc. Amer. Math. Soc. 58 (1976), 197-200.

[3] M.M. Day, Normed linear spaces 3rd ed. (Springer-Verlag, Berlin, Heidelberg, New York, 1973).

[4] F.S. de Blasi, 'On a property of the unit sphere in a Banach Space', Bull. Math. Soc. Sci. Math. R.S. Roumaine (N.S.) 21 (1977), 259-262.

[5] J.R. Giles, D.A. Gregory and B. Sims, 'Geometrical implications of upper semi-continuity of the duality mapping on a Banach space', Pacific J. Math. 79 (1978), 99-109.

[6] J.R. Giles and D.N. Kutzarova, 'Characterisation of drop and weak drop properties for closed bounded convex sets', Bull. Austral. Math. Soc. 43 (1991), 377-385.

[7] J.R. Giles and W.B. Moors, 'A continuity property related to Kuratowski's index of non-compactness, its relevance to the drop property and its implications for differentiability theory', J. Math. Anal. Appl. (to appear).

[8] L. Jokl, 'Minimal convex valued weak' usco correspondences and the Radon-Nikodym property', Comm. Math. Univ. Carolinae 28 (1987), 353-376.

[9] P.S. Kenderov and J.R. Giles, 'On the structure of Banach spaces with Mazur's intersection property', Math. Ann. 291 (1991), 463-473.

[10] D.N. Kutzarova, 'On the drop property of convex sets in Banach spaces', in Constructive theory of functions, 1987 (Sofia, 1988), pp. 283-287.

[11] D.N. Kutzarova and P.L. Papini, 'On some properties concerning convex sets and weak convergence'. Preprint .

[12] I. Namioka, 'Separate continuity and joint continuity', Pacific J. Math. 51 (1974), 515-531.

[13] I. Namioka and R.R. Phelps, 'Banach spaces which are Asplund spaces', Duke Math. J. 42 (1975), 735-750.

[14] R.R. Phelps, Convex functions, monotone operators and differentiability, Lecture Notes in Mathematics 1364 (Springer-Verlag, Berlin, Heidelberg, New York, 1989).

[15] R.R. Phelps, 'Weak* support poins of convex sets in $E^{*}$, Israel J. Math. 2 (1964), 177-182.

[16] S.L. Troyanski, 'On a property of the norm which is close to local uniform rotundity', Math. Ann. 271 (1985), 305-314.

[17] S.L. Troyanski, 'On some generalisations of denting points'. Preprint . 
Department of Mathematics The University of Newcastle New South Wales 2308

Australia 